\title{
Characterizations of Some Classes of Perfect Spaces in Terms of Continuous Selections Avoiding Supporting Sets by
}

\author{
Takamitsu YAMAUCHI
}

Presented by Czestaw BESSAGA

\begin{abstract}
Summary. Some kinds of perfect spaces, including paracompact perfectly normal spaces and collectionwise normal perfect spaces, are characterized in terms of continuous selections avoiding supporting sets. A necessary and sufficient condition on a domain space for a selection theorem of E. Michael [Fund. Math. 47 (1959), 173-178] to hold is also obtained.
\end{abstract}

1. Introduction. Throughout this paper, all spaces are assumed to be $T_{1}$-spaces. Let $2^{Y}$ denote the set of all non-empty subsets of a space $Y$. For a mapping $\varphi: X \rightarrow 2^{Y}$, a mapping $f: X \rightarrow Y$ is called a selection of $\varphi$ if $f(x) \in \varphi(x)$ for each $x \in X$. In [7], E. Michael established theorems on continuous selections for convex-valued mappings, which characterize several topological properties such as paracompactness, normality, collectionwise normality and perfect normality. In most of the theorems, set-valued mappings are assumed to be closed-valued. On the other hand, Theorem 3.1"I in [7] characterizing perfect normality ensures the existence of continuous selections for some set-valued mappings with non-closed values. An essential part of the proof is to obtain a continuous selection avoiding supporting sets of each value for a closed and convex-valued mapping.

The set of all non-empty closed convex subsets of a Banach space $Y$ is denoted by $\mathcal{F}_{c}(Y)$. For $K \in \mathcal{F}_{c}(Y)$, a supporting set $S$ of $K$ is a closed convex proper subset of $K$ such that if an interior point of a segment in $K$ is contained in $S$, then the whole segment lies in $S$. For example, supporting sets of finite-dimensional simplexes are their proper faces. Let $I(K)$ denote

2000 Mathematics Subject Classification: 54C60, 54C65, 54D15.

Key words and phrases: set-valued mapping, selection, supporting set, perfectly normal. 
the set of all points of $K$ which are not in any supporting set of $K$. A mapping $\varphi: X \rightarrow 2^{Y}$ is called lower semicontinuous (l.s.c. for short) if for every open subset $V$ of $Y$, the set $\varphi^{-1}[V]=\{x \in X \mid \varphi(x) \cap V \neq \emptyset\}$ is open in $X$. A topological (respectively, normal) space is said to be perfect (respectively, perfectly normal) if every open subset is an $F_{\sigma}$-set. Theorem $3.1^{\prime \prime \prime}$ in [7] can be restated as follows.

TheOREM 1.1 (E. Michael [7]). A $T_{1}$-space $X$ is perfectly normal if and only if for every separable Banach space $Y$, every l.s.c. mapping $\varphi: X \rightarrow$ $\mathcal{F}_{c}(Y)$ admits a continuous selection $f: X \rightarrow Y$ such that $f(x) \in I(\varphi(x))$ for each $x \in X$.

In [8], E. Michael proved a theorem on dense families of continuous selections, and extended the "only if" part of Theorem 1.1 to arbitrary Banach spaces, but for more restrictive domains.

Theorem 1.2 (E. Michael [8, Corollary 1.3]). If $X$ is a metrizable space, then for every Banach space $Y$, every l.s.c. mapping $\varphi: X \rightarrow \mathcal{F}_{c}(Y)$ admits a continuous selection $f: X \rightarrow Y$ such that $f(x) \in I(\varphi(x))$ whenever $\varphi(x)$ is separable.

As in [7], several topological properties of domain spaces $X$ have been characterized in terms of selections (see also [12], [13]). From this point of view, Theorem 1.2 suggests the possibility of characterizing a certain class of perfectly normal spaces. The purpose of this paper is to obtain characterizations of some classes of perfect spaces in terms of selections avoiding supporting sets.

In Section 2, we consider set-valued mappings with compact values, and prove characterizations of paracompact perfectly normal spaces and collectionwise normal perfect spaces in terms of continuous selections.

In Section 3, we obtain a necessary and sufficient condition on $X$ for the conclusion of Theorem 1.2 to hold. The first infinite ordinal number is denoted by $\omega$. For a space $X$, a cover $\mathcal{U}$ of $X$ and $x \in X$, let $\operatorname{ord}_{x} \mathcal{U}=$ $\operatorname{Card}\{U \in \mathcal{U} \mid x \in U\}$, where Card stands for cardinality. Let us say that a space $X$ has property $(S)$ if for every open cover $\mathcal{U}$ of $X$, there exists a $\sigma$-discrete collection $\mathcal{F}$ of closed subsets of $X$ such that if $x \in U \in \mathcal{U}$ and $\operatorname{ord}_{x} \mathcal{U} \leq \omega$, then $x \in F \subset U$ for some $F \in \mathcal{F}$. This property is suggested by a characterization of perfect spaces due to J. M. Worrell, Jr. and H. H. Wicke [17] (see Lemma 2.3). We prove the following.

THeOREM 1.3. A $T_{1}$-space $X$ is a paracompact Hausdorff space satisfying $(S)$ if and only if for every Banach space $Y$, every mapping $\varphi: X \rightarrow \mathcal{F}_{c}(Y)$ admits a continuous selection $f: X \rightarrow Y$ such that $f(x) \in I(\varphi(x))$ whenever $\varphi(x)$ is separable. 
Note that the Sorgenfrey line is a non-metrizable paracompact Hausdorff space satisfying $(S)$ (see Remark 3.8). An analogous result for separablevalued mappings is also obtained in Section 3.

In this paper, we use the following notation and terminology. The set of all positive integers and the set of all real numbers are denoted by $\mathbb{N}$ and $\mathbb{R}$, respectively. An ordinal number is the set of smaller ordinal numbers, and a cardinal number is an initial ordinal number. The first uncountable ordinal number is denoted by $\omega_{1}$. Throughout this paper, $\gamma$ stands for an infinite cardinal number. The closure of a subset $A$ is denoted by $\mathrm{Cl} A$. The weight of a space $Y$ is denoted by $w(Y)$. An indexed collection $\left\{U_{\lambda} \mid \lambda \in \Lambda\right\}$ of subsets of a space $X$ is point-countable if for each $x \in X$ the set $\left\{\lambda \in \Lambda \mid x \in U_{\lambda}\right\}$ is countable, while $\left\{U_{\lambda} \mid \lambda \in \Lambda\right\}$ is called discrete if each $x \in X$ has a neighborhood $N$ such that $\operatorname{Card}\left\{\lambda \in \Lambda \mid U_{\lambda} \cap N \neq \emptyset\right\} \leq 1$. For a Banach space $(Y,\|\cdot\|), y \in Y$ and $r>0$, let $B(y, r)=\{z \in Y \mid$ $\|y-z\|<r\}$. The convex hull of a subset $A$ of a Banach space is denoted by conv $A$. For a mapping $\varphi: X \rightarrow 2^{Y}$ and a collection $\mathcal{V}$ of subsets of $Y$, let $\varphi^{-1}[\mathcal{V}]=\left\{\varphi^{-1}[V] \mid V \in \mathcal{V}\right\}$. For undefined notation and terminology, we refer to [1].

2. Theorems for mappings with compact values. In this section, we obtain characterizations of some kinds of perfect spaces in terms of mappings with compact values. The following lemma was essentially proved by E. Michael (see the proof of [7, Theorem 3.1'”]).

Lemma 2.1 (E. Michael [7]). Let $X$ be a topological space, $Y$ a Banach space and $\varphi: X \rightarrow \mathcal{F}_{c}(Y)$ a mapping. If there is a countable collection $\left\{f_{i} \mid i \in \mathbb{N}\right\}$ of continuous selections $f_{i}: X \rightarrow Y$ of $\varphi$, then there exists a continuous selection $f: X \rightarrow Y$ of $\varphi$ such that $f(x) \in I(\varphi(x))$ whenever $\left\{f_{i}(x) \mid i \in \mathbb{N}\right\}$ is dense in $\varphi(x)$.

Thus, a key to obtaining a continuous selection that avoids supporting sets is to construct a sequence of continuous selections as in Lemma 2.1. For a mapping $\varphi: X \rightarrow 2^{Y}$, a mapping $\phi: X \rightarrow 2^{Y}$ is called a set-valued selection of $\varphi$ if $\phi(x) \subset \varphi(x)$ for each $x \in X$. For l.s.c. set-valued selections, we have the following.

Lemma 2.2. Let $X$ be a perfect space, $Y$ a Banach space, $\varphi: X \rightarrow \mathcal{F}_{c}(Y)$ an l.s.c. mapping and $A$ an $F_{\sigma}$-set of $X$ such that $\{x \in X \mid \varphi(x)$ is compact $\}$ $\subset A$. Then there exists a sequence $\left\{\phi_{i} \mid i \in \mathbb{N}\right\}$ of l.s.c. set-valued selections $\phi_{i}: X \rightarrow \mathcal{F}_{c}(Y)$ of $\varphi$ such that $\phi_{i}(x)=\varphi(x)$ for each $x \in X \backslash A$ and $i \in \mathbb{N}$, and for each $x \in X$ with $\varphi(x)$ compact, every open subset $W$ of $Y$ with $\varphi(x) \cap W \neq \emptyset$ contains $\phi_{i}(x)$ for some $i \in \mathbb{N}$. 
To prove Lemma 2.2, we need the following lemma which was essentially proved by J. M. Worrell, Jr. and H. H. Wicke [17] (see also [11, Theorem 3]).

Lemma 2.3 (J. M. Worrell and H. H. Wicke [17, Lemma]). For every open cover $\mathcal{U}$ of a perfect space $X$, there exists a $\sigma$-discrete collection $\mathcal{F}$ of closed subsets of $X$ such that if $x \in U \in \mathcal{U}$ and $\operatorname{ord}_{x} \mathcal{U}<\omega$, then $x \in F \subset U$ for some $F \in \mathcal{F}$.

Proof of Lemma 2.2. Our proof is based on the idea used by E. Michael [8]. Put $X^{\prime}=\{x \in X \mid \varphi(x)$ is compact $\}$. Take a sequence $\left\{\mathcal{V}_{i} \mid i \in \mathbb{N}\right\}$ of locally finite open covers of $Y$ such that mesh $\mathcal{V}_{i}<1 / 2^{i}$. Then $X^{\prime} \subset$ $\left\{x \in X \mid \operatorname{ord}_{x} \varphi^{-1}\left[\mathcal{V}_{i}\right]<\omega\right\}$ for each $i \in \mathbb{N}$. By Lemma 2.3, for each $i \in \mathbb{N}$, there exists a $\sigma$-discrete collection $\mathcal{F}_{i}$ of closed subsets of $X$ such that if $x \in X^{\prime}$ and $V \in \mathcal{V}_{i}$ with $x \in \varphi^{-1}[V]$, then $x \in F \subset \varphi^{-1}[V]$ for some $F \in \mathcal{F}_{i}$. We may assume that $\bigcup \mathcal{F}_{i} \subset A$ and $X^{\prime} \cap F \neq \emptyset$ for each $F \in \mathcal{F}_{i}$. For $i \in \mathbb{N}$ and $F \in \mathcal{F}_{i}$, the set $\left\{V \in \mathcal{V}_{i} \mid F \subset \varphi^{-1}[V]\right\}$ is finite, say $\left\{V_{k}^{F} \mid k=1, \ldots, k_{F}\right\}$. For $F \in \bigcup_{i \in \mathbb{N}} \mathcal{F}_{i}$ and $k \in\left\{1, \ldots, k_{F}\right\}$, define $\phi_{k}^{F}$ : $F \rightarrow \mathcal{F}_{c}(Y)$ by putting $\phi_{k}^{F}(x)=\mathrm{Cl}\left(\operatorname{conv}\left(\varphi(x) \cap V_{k}^{F}\right)\right)$ for each $x \in F$. Then $\phi_{k}^{F}$ is l.s.c. (see [7, Propositions 2.3, 2.4 and 2.6]). For each $i \in \mathbb{N}$, take discrete collections $\mathcal{F}_{(i, j)}, j \in \mathbb{N}$, of closed subsets of $X$ such that $\mathcal{F}_{i}=$ $\bigcup_{j \in \mathbb{N}} \mathcal{F}_{(i, j)}$. For $i, j, k \in \mathbb{N}$, define $\phi_{(i, j, k)}: X \rightarrow \mathcal{F}_{c}(Y)$ by $\phi_{(i, j, k)}(x)=\phi_{k}^{F}(x)$ if $x \in F \in \mathcal{F}_{(i, j)}$ and $k \leq k_{F}$, and $\phi_{(i, j, k)}(x)=\varphi(x)$ otherwise. Then the sequence $\left\{\phi_{(i, j, k)} \mid i, j, k \in \mathbb{N}\right\}$ is as required.

The following lemma is easy to verify and we omit the proof.

Lemma 2.4. Let $K$ be a closed convex subset of a Banach space $Y$. If $V$ is an open convex subset of $Y$, then $I(\mathrm{Cl}(K \cap V)) \subset I(K)$. If $C_{n} \subset I(K)$ for each $n=1, \ldots, k$, then conv $\bigcup\left\{C_{n} \mid n=1, \ldots, k\right\} \subset I(K)$.

We have the following characterization of perfect spaces.

TheOREM 2.5. For a topological space $X$, the following statements are equivalent.

(a) $X$ is perfect.

(b) For every Banach space $Y$, every l.s.c. mapping $\varphi: X \rightarrow \mathcal{F}_{c}(Y)$ admits an l.s.c. set-valued selection $\phi: X \rightarrow \mathcal{F}_{c}(Y)$ such that $\phi(x) \subset$ $I(\varphi(x))$ whenever $\varphi(x)$ is compact.

(c) Every l.s.c. mapping $\varphi: X \rightarrow \mathcal{C}_{c}(\mathbb{R})$ admits an l.s.c. set-valued selection $\phi: X \rightarrow \mathcal{C}_{c}(\mathbb{R})$ such that $\phi(x) \subset I(\varphi(x))$ for each $x \in X$.

Proof. (a) $\Rightarrow$ (b). Fix $n \in \mathbb{N}$. Put $U_{n}=\varphi^{-1}[B(0, n)]$ and define $\varphi_{n}$ : $U_{n} \rightarrow \mathcal{F}_{c}(Y)$ by $\varphi_{n}(x)=\mathrm{Cl}(\varphi(x) \cap B(0, n))$ for each $x \in X$. Take a sequence $\left\{\theta_{i}^{n} \mid i \in \mathbb{N}\right\}$ of l.s.c. set-valued selections $\theta_{i}^{n}: U_{n} \rightarrow \mathcal{F}_{c}(Y)$ of $\varphi_{n}$ as in Lemma 2.2. Define $\theta_{n}: U_{n} \rightarrow 2^{Y}$ by $\theta_{n}(x)=\left\{\sum_{i=1}^{\infty}(1 / 2)^{i} y_{i} \mid y_{i} \in\right.$ $\left.\theta_{i}^{n}(x), i \in \mathbb{N}\right\}$ for each $x \in X$. Since $\varphi_{n}(x)$ is bounded and complete, the 
limit $\sum_{i=1}^{\infty}(1 / 2)^{i} y_{i}$ always exists. The mapping $s: \varphi_{n}(x)^{\mathbb{N}} \rightarrow \varphi_{n}(x)$ defined by $s\left(\left(y_{i}\right)_{i \in \mathbb{N}}\right)=\sum_{i=1}^{\infty}(1 / 2)^{i} y_{i}$ for each $\left(y_{i}\right)_{i \in \mathbb{N}} \in \varphi_{n}(x)^{\mathbb{N}}$, where $\varphi_{n}(x)^{\mathbb{N}}$ is endowed with the product topology, is continuous. Thus $\theta_{n}$ is l.s.c., and $\theta_{n}(x)$ is compact whenever $\varphi(x)$ is compact. Since each $\theta_{i}^{n}$ is convex-valued, so is $\theta_{n}$. If $\varphi(x)$ is compact and $y_{i} \in \theta_{i}^{n}(x)$ for each $i \in \mathbb{N}$, then the set $\left\{y_{i} \mid i \in \mathbb{N}\right\}$ is dense in $\varphi_{n}(x)$. By Lemma 2.4 and the same argument as in [7, Lemma 5.1], we have $\theta_{n}(x) \subset I\left(\varphi_{n}(x)\right)=I(\mathrm{Cl}(\varphi(x) \cap B(0, n))) \subset I(\varphi(x))$ whenever $\varphi(x)$ is compact. Thus the mapping $\phi_{n}: U_{n} \rightarrow \mathcal{F}_{c}(Y)$ defined by $\phi_{n}(x)=\operatorname{Cl}\left(\theta_{n}(x)\right)$ is an 1.s.c. set-valued selection of $\left.\varphi\right|_{U_{n}}$ such that $\phi_{n}(x) \subset I(\varphi(x))$ whenever $\varphi(x)$ is compact.

Here, $\left\{U_{n} \mid n \in \mathbb{N}\right\}$ is an open cover of the perfect space $X$ such that $U_{n} \subset U_{n+1}$ for each $n \in \mathbb{N}$. Thus each open set $U_{n}$ can be written as $U_{n}=\bigcup_{i \in \mathbb{N}} E_{i}^{n}$, where $E_{i}^{n}, i \in \mathbb{N}$, are closed subsets of $X$. Put $F_{0}=\emptyset$ and $F_{n}=\bigcup_{i \leq n} E_{i}^{i}$ for each $n \in \mathbb{N}$. Then $\left\{F_{n} \mid n \in \mathbb{N}\right\}$ is a closed cover of $X$ such that $F_{n} \subset U_{n}$ and $F_{n} \subset F_{n+1}$ for each $n \in \mathbb{N}$, and hence $\left\{U_{n} \backslash F_{n-1} \mid n \in \mathbb{N}\right\}$ is a point-finite open cover of $X$. Define $\phi: X \rightarrow \mathcal{F}_{c}(Y)$ by $\phi(x)=\mathrm{Cl}\left(\operatorname{conv} \bigcup\left\{\phi_{n}(x) \mid x \in U_{n} \backslash F_{n-1}\right\}\right)$. Then $\phi$ is an l.s.c. set-valued selection of $\varphi$. If $\varphi(x)$ is compact, then conv $\bigcup\left\{\phi_{n}(x) \mid x \in U_{n} \backslash F_{n-1}\right\}$ is the convex hull of the union of finitely many compact convex subsets of $Y$, and hence it is compact (cf. [6, Lemma 2.10.14]). Thus, by Lemma 2.4, $\phi(x) \subset I(\varphi(x))$ whenever $\varphi(x)$ is compact.

(b) $\Rightarrow(\mathrm{c})$. This is obvious.

(c) $\Rightarrow$ (a). Assume that $X$ satisfies (c) and let $U$ be an open subset of $X$. Define $\varphi: X \rightarrow 2^{\mathbb{R}}$ by $\varphi(x)=[0,1]$ if $x \in U$ and $\varphi(x)=\{0\}$ otherwise. Then $\varphi: X \rightarrow \mathcal{C}_{c}(\mathbb{R})$ is l.s.c. and, by (c), there exists an l.s.c. mapping $\phi: X \rightarrow \mathcal{C}_{c}(\mathbb{R})$ such that $\phi(x) \subset I(\varphi(x))$. Put $F_{n}=X \backslash \phi^{-1}[(-1,1 / n)]$ for each $n \in \mathbb{N}$. Then each $F_{n}$ is closed in $X$. Since $I([0,1])=(0,1)$, we have $U=\bigcup_{n \in \mathbb{N}} F_{n}$.

A space $X$ is $\gamma$-paracompact if every open cover $\mathcal{U}$ of $X$ with Card $\mathcal{U} \leq \gamma$ is refined by a locally finite open cover of $X$.

TheOREM 2.6. For a $T_{1}$-space $X$, the following statements are equivalent.

(a) $X$ is $\gamma$-paracompact and perfectly normal.

(b) For every Banach space $Y$ with $w(Y) \leq \gamma$, every l.s.c. mapping $\varphi$ : $X \rightarrow \mathcal{F}_{c}(Y)$ admits a sequence $\left\{f_{i} \mid i \in \mathbb{N}\right\}$ of continuous selections $f_{i}: X \rightarrow Y$ such that $\left\{f_{i}(x) \mid i \in \mathbb{N}\right\}$ is dense in $\varphi(x)$ whenever $\varphi(x)$ is compact.

(c) For every Banach space $Y$ with $w(Y) \leq \gamma$, every l.s.c. mapping $\varphi$ : $X \rightarrow \mathcal{F}_{c}(Y)$ admits a continuous selection $f: X \rightarrow Y$ such that $f(x) \in I(\varphi(x))$ whenever $\varphi(x)$ is compact. 
Proof. To show $(\mathrm{a}) \Rightarrow(\mathrm{b})$, let $X$ be a $\gamma$-paracompact perfectly normal space, $Y$ a Banach space with $w(Y) \leq \gamma$, and $\varphi: X \rightarrow \mathcal{F}_{c}(Y)$ an l.s.c. mapping. Then there exists a sequence $\left\{\phi_{i} \mid i \in \mathbb{N}\right\}$ of l.s.c. mappings $\phi_{i}$ : $X \rightarrow \mathcal{F}_{c}(Y)$ as in Lemma 2.2. By virtue of [7, Theorem 3.2" $]$ (see also [10, Theorem 4.1]), there exists a continuous selection $f_{i}: X \rightarrow Y$ of $\phi_{i}$ for each $i \in \mathbb{N}$. Then $\left\{f_{i} \mid i \in \mathbb{N}\right\}$ is the required sequence.

The implication $(\mathrm{b}) \Rightarrow(\mathrm{c})$ follows from Lemma 2.1 , and $(\mathrm{c}) \Rightarrow(\mathrm{a})$ follows from [7, Theorem 3.2"] (see also [10, Theorem 4.1]) and Theorem 2.5.

COROLlary 2.7. A $T_{1}$-space $X$ is paracompact and perfectly normal if and only if for every Banach space $Y$, every l.s.c. mapping $\varphi: X \rightarrow \mathcal{F}_{c}(Y)$ admits a continuous selection $f: X \rightarrow Y$ such that $f(x) \in I(\varphi(x))$ whenever $\varphi(x)$ is compact.

A space $X$ is $\gamma$-collectionwise normal if for every discrete collection $\left\{F_{\lambda} \mid \lambda \in \Lambda\right\}$ of closed subsets of $X$ with Card $\Lambda \leq \gamma$, there exists a disjoint collection $\left\{U_{\lambda} \mid \lambda \in \Lambda\right\}$ of open subsets of $X$ such that $F_{\lambda} \subset U_{\lambda}$ for each $\lambda \in \Lambda$. For a Banach space $Y$, let $\mathcal{C}_{c}(Y)$ be the set of all non-empty compact convex subsets of $Y$ and put $\mathcal{C}_{c}^{\prime}(Y)=\mathcal{C}_{c}(Y) \cup\{Y\}$.

TheOREm 2.8. For a $T_{1}$-space $X$, the following statements are equivalent.

(a) $X$ is $\gamma$-collectionwise normal and perfect.

(b) For every Banach space $Y$ with $w(Y) \leq \gamma$, every l.s.c. mapping $\varphi$ : $X \rightarrow \mathcal{C}_{c}^{\prime}(Y)$ admits a sequence $\left\{f_{i} \mid i \in \mathbb{N}\right\}$ of continuous selections $f_{i}: X \rightarrow Y$ such that $\left\{f_{i}(x) \mid i \in \mathbb{N}\right\}$ is dense in $\varphi(x)$ whenever $\varphi(x)$ is compact.

(c) For every Banach space $Y$ with $w(Y) \leq \gamma$, every l.s.c. mapping $\varphi$ : $X \rightarrow \mathcal{C}_{c}^{\prime}(Y)$ admits a continuous selection $f: X \rightarrow Y$ such that $f(x) \in I(\varphi(x))$ for each $x \in X$.

Proof. To show $(\mathrm{a}) \Rightarrow(\mathrm{b})$, let $X$ be a $\gamma$-collectionwise normal perfect space, $Y$ a Banach space with $w(Y) \leq \gamma$, and $\varphi: X \rightarrow \mathcal{C}_{c}^{\prime}(Y)$ an l.s.c. mapping. Put $A=\left\{x \in X \mid \varphi(x) \in \mathcal{C}_{c}(Y)\right\}$. Then $A$ is an $F_{\sigma}$-set. Thus (b) follows from Lemma 2.2 and [7, Theorem 3.2'] (see also [10, Theorem 4.2]).

The implication $(\mathrm{b}) \Rightarrow(\mathrm{c})$ follows from Lemma 2.1 since if $\varphi(x)$ is not compact, then $\varphi(x)=Y$. The implication (c) $\Rightarrow($ a) follows from [7, Theorem 3.2'] (see also [10, Theorem 4.2]) and Theorem 2.5.

COROllary 2.9. A $T_{1}$-space $X$ is collectionwise normal and perfect if and only if for every Banach space $Y$, every l.s.c. mapping $\varphi: X \rightarrow \mathcal{C}_{c}^{\prime}(Y)$ admits a continuous selection $f: X \rightarrow Y$ such that $f(x) \in I(\varphi(x))$ for each $x \in X$. 
Remark 2.10. For $K \in \mathcal{F}_{c}(Y)$, a point $y \in Y$ is called an extreme point if $K \backslash\{y\}$ is convex. The weak convex interior wci $(K)$ of $K([3])$ is the set of all non-extreme points of $K$. Then wci $(K) \subset I(K)$ for each $K \in \mathcal{F}_{c}(Y)$ with Card $K>1$. Thus the "only if" part of Theorem 2.8 is an extension of $(1) \Rightarrow(2)$ of $[3$, Theorem 4.6] and $(a) \Rightarrow(b)$ of [18, Corollary 10].

A space $X$ is said to be $\gamma$-PF-normal if every point-finite open cover $\mathcal{U}$ of $X$ with $\operatorname{Card} \mathcal{U} \leq \gamma$ is normal. A space is called PF-normal ([15]) if it is $\gamma$-PF-normal for every infinite cardinal number $\gamma$. Note that every $\gamma$ collectionwise normal space is $\gamma$-PF-normal, and $\omega$-PF-normality coincides with normality.

TheOREm 2.11. For a $T_{1}$-space $X$, the following statements are equivalent.

(a) $X$ is $\gamma$-PF-normal and perfect.

(b) For every Banach space $Y$ with $w(Y) \leq \gamma$, every l.s.c. mapping $\varphi$ : $X \rightarrow \mathcal{C}_{c}(Y)$ admits a sequence $\left\{f_{i} \mid i \in \mathbb{N}\right\}$ of continuous selections $f_{i}: X \rightarrow Y$ such that $\left\{f_{i}(x) \mid i \in \mathbb{N}\right\}$ is dense in $\varphi(x)$ for each $x \in X$.

(c) For every Banach space $Y$ with $w(Y) \leq \gamma$, every l.s.c. mapping $\varphi$ : $X \rightarrow \mathcal{C}_{c}(Y)$ admits a continuous selection such that $f(x) \in I(\varphi(x))$ for each $x \in X$.

Proof. The implication (a) $\Rightarrow$ (b) follows from Lemma 2.2 and [10, Theorem 4.1] (note that, in the realm of normal spaces, $\gamma$-PF-normality coincides with $\gamma$-pointwise- $\omega$-paracompactness of [10]). The implication (b) $\Rightarrow(\mathrm{c})$ follows from Lemma 2.1, and (c) $\Rightarrow$ (a) follows from [10, Theorem 4.1] and Theorem 2.5.

Corollary 2.12. A $T_{1}$-space $X$ is PF-normal and perfect if and only if for every Banach space $Y$, every l.s.c. mapping $\varphi: X \rightarrow \mathcal{C}_{c}(Y)$ admits a continuous selection such that $f(x) \in I(\varphi(x))$ for each $x \in X$.

3. Theorems for mappings with separable values. First, we prove Theorem 1.3. Let us consider the following property for a space $X$.

$\left(S_{\gamma}\right)$ For every open cover $\mathcal{U}$ of $X$ with $\operatorname{Card} \mathcal{U} \leq \gamma$, there exists a $\sigma$ discrete collection $\mathcal{F}$ of closed subsets of $X$ such that if $x \in U \in \mathcal{U}$ and $\operatorname{ord}_{x} \mathcal{U} \leq \omega$, then $x \in F \subset U$ for some $F \in \mathcal{F}$.

A space satisfies $(S)$ if it satisfies $\left(S_{\gamma}\right)$ for every infinite cardinal $\gamma$. Note that a space satisfies $\left(S_{\omega}\right)$ if and only if it is perfect. It is shown in Remark 3.8 that a perfect space need not satisfy $(S)$. Theorem 1.3 follows from the following. 
TheOREM 3.1. For a $T_{1}$-space $X$, the following statements are equivalent.

(a) $X$ is a normal $\gamma$-paracompact space satisfying $\left(S_{\gamma}\right)$.

(b) For every Banach space $Y$ with $w(Y) \leq \gamma$, every l.s.c. mapping $\varphi$ : $X \rightarrow \mathcal{F}_{c}(Y)$ admits a sequence $\left\{f_{i} \mid i \in \mathbb{N}\right\}$ of continuous selections $f_{i}: X \rightarrow Y$ such that $\left\{f_{i}(x) \mid i \in \mathbb{N}\right\}$ is dense in $\varphi(x)$ whenever $\varphi(x)$ is separable.

(c) For every Banach space $Y$ with $w(Y) \leq \gamma$, every l.s.c. mapping $\varphi$ : $X \rightarrow \mathcal{F}_{c}(Y)$ admits a continuous selection $f: X \rightarrow Y$ such that $f(x) \in I(\varphi(x))$ whenever $\varphi(x)$ is separable.

Proof. The implication $(\mathrm{a}) \Rightarrow$ (b) is obtained by repeating the proof of [8, Theorem 1.1], and (b) $\Rightarrow$ (c) follows from Lemma 2.1.

To show $(\mathrm{c}) \Rightarrow(\mathrm{a})$, let $X$ be a space satisfying (c). By [7, Theorem $\left.3.2^{\prime \prime}\right]$ (see also [10, Theorem 4.1]), $X$ is normal and $\gamma$-paracompact. To show that it satisfies $\left(S_{\gamma}\right)$, let $\mathcal{U}$ be an open cover of $X$ with $\operatorname{Card} \mathcal{U} \leq \gamma$. For any set $\Lambda$, let $l_{1}(\Lambda)$ be the usual Banach space of summable functions $s: \Lambda \rightarrow \mathbb{R}$ with the norm $\|s\|=\sum_{\lambda \in \Lambda}|s(\lambda)|$. Following [7], define an l.s.c. mapping $\varphi: X \rightarrow$ $\mathcal{F}_{c}\left(l_{1}(\mathcal{U})\right)$ by $\varphi(x)=\left\{y \in l_{1}(\mathcal{U}) \mid\|y\|=1, y(U) \geq 0\right.$ for every $U \in \mathcal{U}$, and $y(U)=0$ for all $U \in \mathcal{U}$ with $x \notin U\}$ for $x \in X$. Here $w\left(l_{1}(\mathcal{U})\right) \leq \gamma$. By $(\mathrm{c})$, there exists a continuous selection $f: X \rightarrow l_{1}(\mathcal{U})$ of $\varphi$ such that $f(x) \in$ $I(\varphi(x))$ whenever $\varphi(x)$ is separable. Let $\mathcal{B}$ be a $\sigma$-discrete base for $l_{1}(\mathcal{U})$.

We claim that the $\sigma$-discrete closed collection $\left\{f^{-1}(\mathrm{Cl} B) \mid B \in \mathcal{B}\right\}$ satisfies the condition of $\left(S_{\gamma}\right)$. Indeed, let $x \in X$ with $\operatorname{ord}_{x} \mathcal{U} \leq \omega$ and $x \in U \in \mathcal{U}$. Then $\varphi(x)$ is separable. Since $S=\{y \in \varphi(x) \mid y(U)=0\}$ is a supporting set of $\varphi(x)$, we have $f(x) \notin S$ and hence $f(x)(U)>0$. Take $B \in \mathcal{B}$ such that $f(x) \in B$ and $\operatorname{diam} B<f(x)(U)$. For each $z \in f^{-1}(\mathrm{Cl} B)$, $f(z)(U) \geq f(x)(U)-|f(x)(U)-f(z)(U)| \geq f(x)(U)-\|f(x)-f(z)\|>0$, and hence $z \in U$. Thus $x \in f^{-1}(\mathrm{Cl} B) \subset U$.

The following example shows that, if a closed convex subset $K$ of a Banach space is not separable, then $I(K)$ may be empty. Thus the separability of values of a set-valued mapping is necessary in order that a selection may avoid all supporting sets of its values.

EXAMPle 3.2. Let $\Lambda$ be an uncountable set and put $K=\left\{s \in l_{1}(\Lambda)\right.$ $s(\lambda) \geq 0$ for each $\lambda \in \Lambda\}$. Take $s \in K$ arbitrary. Then there exists $\lambda_{s} \in \Lambda$ such that $s\left(\lambda_{s}\right)=0$ and the set $S=\left\{t \in K \mid t\left(\lambda_{s}\right)=0\right\}$ is a supporting set of $K$ containing $s$. Thus $I(K)=\emptyset$.

Next, we consider continuous selections avoiding supporting sets for separable-valued mappings. For a Banach space $Y$, let $\mathcal{S}_{c}(Y)=\left\{S \in \mathcal{F}_{c}(Y) \mid\right.$ $S$ is separable\}. Mimicking the definition of $\gamma$-PF-normality, let us call a 
space $X \gamma$-PC-normal if every point-countable open cover $\mathcal{U}$ of $X$ with Card $\mathcal{U} \leq \gamma$ is normal. A space is called $P C$-normal if it is $\gamma$-PC-normal for every infinite cardinal number $\gamma$. Note that every $\gamma$-PC-normal space is countably paracompact and normal. For the same reason as in [3, p. 506], $\gamma$-PC-normality is not hereditary with respect to closed subsets in general. But $\gamma$-PC-normality is hereditary with respect to open $F_{\sigma}$-sets. The proof is quite analogous to the proof that $\gamma$-PF-normality is hereditary with respect to open $F_{\sigma}$-sets (cf. [19]).

For collections $\mathcal{U}$ and $\mathcal{F}$ of subsets of a space $X, \mathcal{F}$ is called a base [4] of $\mathcal{U}$ if each member of $\mathcal{U}$ is a union of members from $\mathcal{F}$. A subset $A$ of a topological space $X$ is called a cozero-set if there is a continuous function $f: X \rightarrow \mathbb{R}$ such that $A=\{x \in X \mid f(x) \neq 0\}$. A base is called closed (respectively, cozero-set) if it consists of closed subsets (respectively, cozerosets). By Lemma 2.3, a space $X$ is perfect if and only if every point-finite open cover of $X$ has a $\sigma$-discrete closed base. Inspired by this characterization, let us consider the following properties of a space $X$.

$\left(S_{\gamma}^{\prime}\right)$ Every point-countable open cover $\mathcal{U}$ with $\operatorname{Card} \mathcal{U} \leq \gamma$ of $X$ has a $\sigma$-discrete closed base.

$\left(S^{\prime}\right) X$ satisfies $\left(S_{\gamma}^{\prime}\right)$ for every infinite cardinal $\gamma$.

Clearly, $\left(S_{\gamma}\right)$ implies $\left(S_{\gamma}^{\prime}\right)$, and a space satisfies $\left(S_{\omega}\right)$ if and only if it is perfect. It is shown in Remark 3.8 that $\left(S^{\prime}\right)$ coincides with neither $(S)$ nor perfectness. For separable-valued mappings, we have the following.

TheOREM 3.3. For a $T_{1}$-space $X$, the following statements are equivalent.

(a) $X$ is a $\gamma$-PC-normal space with $\left(S_{\gamma}^{\prime}\right)$.

(b) Every point-countable open cover $\mathcal{U}$ of $X$ with $\operatorname{Card} \mathcal{U} \leq \gamma$ has a $\sigma$-discrete cozero-set base.

(c) For every Banach space $Y$ with $w(Y) \leq \gamma$, every l.s.c. mapping $\varphi$ : $X \rightarrow \mathcal{S}_{c}(Y)$ admits a sequence $\left\{f_{i} \mid i \in \mathbb{N}\right\}$ of continuous selections $f_{i}: X \rightarrow Y$ such that $\left\{f_{i}(x) \mid i \in \mathbb{N}\right\}$ is dense in $\varphi(x)$.

(d) For every Banach space $Y$ with $w(Y) \leq \gamma$, every l.s.c. mapping $\varphi$ : $X \rightarrow \mathcal{S}_{c}(Y)$ admits a continuous selection $f: X \rightarrow Y$ such that $f(x) \in I(\varphi(x))$ for each $x \in X$.

To prove Theorem 3.3, we need some preparation. S. Nedev [10] proved the following selection theorem for $\gamma$-PC-normal spaces (note that, in the realm of normal spaces, $\gamma$-PC-normality coincides with $\gamma$-pointwise- $\omega_{1}$-paracompactness of [10]).

Theorem 3.4 (S. Nedev [10, Theorem 4.1]). A $T_{1}$-space $X$ is $\gamma$-PCnormal if and only if for every Banach space $Y$ with $w(Y) \leq \gamma$, every l.s.c. mapping $\varphi: X \rightarrow \mathcal{S}_{c}(Y)$ admits a continuous selection. 
For a collection $\mathcal{F}=\left\{F_{\lambda} \mid \lambda \in \Lambda\right\}$ of subsets of a space $X$, another such collection $\left\{U_{\lambda} \mid \lambda \in \Lambda\right\}$ is called an expansion of $\mathcal{F}$ if $F_{\lambda} \subset U_{\lambda}$ for each $\lambda \in \Lambda$. An expansion is called open if it consists of open subsets. For a subset $A$ of a space $X$ and a collection $\mathcal{U}$ of subsets of $X$, let $\operatorname{St}(A, \mathcal{U})=$ $\bigcup\{U \in \mathcal{U} \mid A \cap U \neq \emptyset\}$.

Lemma 3.5. If $X$ is $\gamma$-PC-normal, then every discrete collection $\left\{F_{\lambda} \mid\right.$ $\lambda \in \Lambda\}$ of closed subsets of $X$ with Card $\Lambda \leq \gamma$ has a discrete open expansion provided it has a point-countable open expansion $\left\{U_{\lambda} \mid \lambda \in \Lambda\right\}$.

Proof. Let $X,\left\{F_{\lambda} \mid \lambda \in \Lambda\right\}$ and $\left\{U_{\lambda} \mid \lambda \in \Lambda\right\}$ be as in the statement. For each $\lambda \in \Lambda$, put $G_{\lambda}=U_{\lambda} \backslash \bigcup_{\mu \neq \lambda, \mu \in \lambda} F_{\mu}$. Then $F_{\lambda} \subset G_{\lambda}$ for each $\lambda \in \Lambda$ and $\mathcal{G}=\left\{G_{\lambda} \mid \lambda \in \Lambda\right\} \cup\left\{X \backslash \bigcup_{\mu \in \Lambda} F_{\mu}\right\}$ is a point-countable open cover of $X$ such that $\operatorname{Card} \mathcal{G} \leq \gamma$, and hence $\mathcal{G}$ is normal. Thus there exists a normal sequence $\left\{\mathcal{G}_{i} \mid i \in \mathbb{N}\right\}$ of open covers of $X$ such that $\mathcal{G}_{1}$ refines $\mathcal{G}$. Then $\left\{\operatorname{St}\left(G, \mathcal{G}_{2}\right) \mid G \in \mathcal{G}_{2}\right\}$ refines $\mathcal{G}$. Thus $\left\{\operatorname{St}\left(F_{\lambda}, \mathcal{G}_{2}\right) \mid \lambda \in \Lambda\right\}$ is a discrete open expansion of $\left\{F_{\lambda} \mid \lambda \in \Lambda\right\}$.

Let us prove Theorem 3.3.

Proof of Theorem 3.3. (a) $\Rightarrow(\mathrm{b})$. Assume (a) and let $\mathcal{U}$ be a point-countable open cover of $X$ with $\operatorname{Card} \mathcal{U} \leq \gamma$. Take a $\sigma$-discrete closed base $\mathcal{F}=\bigcup_{i \in \mathbb{N}} \mathcal{F}_{i}$ of $\mathcal{U}$, where $\mathcal{F}_{i}$ is discrete. We may assume $\mathcal{F}$ refines $\mathcal{U}$ and each $F \in \mathcal{F}$ is non-empty. For each $F \in \mathcal{F}$, the set $\{U \in \mathcal{U} \mid F \subset U\}$ is countable, say $\{U(F, j) \mid j \in \mathbb{N}\}$. Fix $i, j \in \mathbb{N}$ and put $\mathcal{U}_{(i, j)}=$ $\left\{U(F, j) \mid F \in \mathcal{F}_{i}\right\}$. For each $U \in \mathcal{U}_{(i, j)}$, put $E_{U}=\bigcup\left\{F \in \mathcal{F}_{i} \mid U(F, j)=U\right\}$. Then $\bigcup\left\{E_{U} \mid U \in \mathcal{U}_{(i, j)}\right\}=\bigcup \mathcal{F}_{i}$, and $\mathcal{U}_{(i, j)}$ is a point-countable open expansion of the discrete collection $\left\{E_{U} \mid U \in \mathcal{U}_{(i, j)}\right\}$ of closed subsets of $X$ of cardinality $\leq \gamma$. By Lemma 3.5 and the normality of $X$, there exists a discrete collection $\mathcal{V}_{(i, j)}=\left\{V_{U} \mid U \in \mathcal{U}_{(i, j)}\right\}$ of cozero-sets of $X$ such that $E_{U} \subset V_{U} \subset U$ for each $U \in \mathcal{U}_{(i, j)}$. Then $\bigcup\left\{\mathcal{V}_{(i, j)} \mid i, j \in \mathbb{N}\right\}$ is a $\sigma$-discrete cozero-set base of $\mathcal{U}$.

(b) $\Rightarrow(\mathrm{c})$. The proof is analogous to that of [8, Theorem 1.1]. Let $X$ be a space satisfying (b), $Y$ a Banach space with $w(Y) \leq \gamma$, and $\varphi: X \rightarrow \mathcal{S}_{c}(Y)$ an l.s.c. mapping. By [9, Theorem 1.2], $X$ is $\gamma$-PC-normal. Let $\left\{\mathcal{V}_{i} \mid i \in \mathbb{N}\right\}$ be a sequence of locally finite open covers of $Y$ such that mesh $\mathcal{V}_{i}<1 / 2^{i}$ for each $i \in \mathbb{N}$. Then $\varphi^{-1}\left[\mathcal{V}_{i}\right]$ is a point-countable open cover of cardinality $\leq \gamma$ for each $i \in \mathbb{N}$. By (b), there exists a $\sigma$-discrete cozero-set base $\mathcal{W}_{i}=\bigcup_{j \in \mathbb{N}} \mathcal{W}_{(i, j)}$ of $\varphi^{-1}\left[\mathcal{V}_{i}\right]$ for each $i \in \mathbb{N}$, where each $\mathcal{W}_{(i, j)}$ is discrete and each $W \in \mathcal{W}_{i}$ is non-empty. For $i \in \mathbb{N}$ and $W \in \mathcal{W}_{i}$, the set $\left\{V \in \mathcal{V}_{i} \mid W \subset \varphi^{-1}[V]\right\}$ is countable, say $\left\{V_{k}^{W} \mid k \in \mathbb{N}\right\}$. For each $W \in \bigcup_{i \in \mathbb{N}} \mathcal{W}_{i}$ and $k \in \mathbb{N}$, define $\varphi_{k}^{W}: W \rightarrow \mathcal{S}_{c}(Y)$ by $\varphi_{k}^{W}(x)=\mathrm{Cl}\left(\operatorname{conv}\left(\varphi(x) \cap V_{k}^{W}\right)\right)$ for $x \in W$. 
Since $W$ is $\gamma$-PC-normal, by Theorem 3.4, $\varphi_{k}^{W}$ admits a continuous selection $g_{k}^{W}: W \rightarrow Y$.

Each $W \in \bigcup_{i \in \mathbb{N}} \mathcal{W}_{i}$ can be written as $W=\bigcup_{n \in \mathbb{N}} F_{n}^{W}$ for some closed sets $F_{n}^{W}, n \in \mathbb{N}$. Then $\left\{F_{n}^{W} \mid W \in \mathcal{W}_{(i, j)}\right\}$ is a discrete collection of closed subsets for each $i, j, n \in \mathbb{N}$. For $i, j, k, n \in \mathbb{N}$, define $\varphi_{(i, j, k, n)}: X \rightarrow \mathcal{S}_{c}(Y)$ by $\varphi_{(i, j, k, n)}(x)=\left\{g_{k}^{W}(x)\right\}$ if $x \in F_{n}^{W}$ and $W \in \mathcal{W}_{(i, j)}$, and $\varphi_{(i, j, k, n)}(x)=\varphi(x)$ otherwise. Since $\varphi_{(i, j, k, n)}$ is l.s.c., there is a continuous selection $f_{(i, j, k, n)}$ : $X \rightarrow Y$ of $\varphi_{(i, j, k, n)}$. Then $\left\{f_{(i, j, k, n)} \mid i, j, n, k \in \mathbb{N}\right\}$ is the required sequence.

$(\mathrm{c}) \Rightarrow(\mathrm{d})$. This follows from Lemma 2.1.

$(\mathrm{d}) \Rightarrow(\mathrm{a})$. Assume that $X$ satisfies (d). By Theorem 3.4, $X$ is $\gamma$-PCnormal. By the same argument as in the proof of the "only if" part of Theorem 3.1, $X$ satisfies $\left(S_{\gamma}^{\prime}\right)$.

Corollary 3.6. A $T_{1}$-space $X$ is a $P C$-normal space with $\left(S^{\prime}\right)$ if and only if for every Banach space $Y$, every mapping $\varphi: X \rightarrow \mathcal{S}_{c}(Y)$ admits a continuous selection $f: X \rightarrow Y$ such that $f(x) \in I(\varphi(x))$ for each $x \in X$.

REMARK 3.7. Lemma 3.5 is a $\gamma$-PC-normal analogue of $(1) \Rightarrow(7)$ of [3, Theorem 3.1]. In Lemma 3.5, if the phrases " $\gamma$-PC-normal" and "pointcountable" are replaced with " $\gamma$-PF-normal" and "point-finite", respectively, then the converse is also valid by [3, Theorem 3.1]. But the converse of Lemma 3.5 itself does not hold in general since every collectionwise normal space satisfies the condition stated in Lemma 3.5, while every PC-normal space is countably paracompact (see [14]).

REMARK 3.8. A regular space $X$ is called a $\sigma$-space if $X$ has a $\sigma$-discrete closed network (for properties of $\sigma$-spaces, see [2]). The following implications hold:

$$
\sigma \text {-space } \Rightarrow(S) \Rightarrow\left(S^{\prime}\right) \Rightarrow \text { perfect. }
$$

Note that every hereditarily separable perfect space satisfies $(S)$. Thus the Sorgenfrey line satisfies $(S)$. But the Sorgenfrey line is not a $\sigma$-space (in fact, it is not a $\beta$-space [5, Example 4.11]). Thus the first arrow cannot be reversed.

Todorčević [16] constructed an example of a non-metrizable paracompact perfect space $X[\geq]$ with a point-countable base. Note that every space which has a point-countable base and satisfies $\left(S^{\prime}\right)$ is a $\sigma$-space, and it is known that every paracompact $\sigma$-space with a point-countable base is metrizable (cf. [2]). Hence the space $X[\geq]$ shows that the third arrow cannot be reversed.

An example showing that the second arrow cannot be reversed is also obtained by using Todorčević's space $X[\geq]$. A key is the fact that every closed subspace of a space satisfying $(S)$ satisfies $(S)$. Notice that $w(X[\geq])$ $=\omega_{1}$, since Card $X[\geq]=\omega_{1}$ by the construction and $X[\geq]$ has a pointcountable base. Thus, we may assume that $X[\geq]$ is a subspace of the product 
$H=[0,1]^{\omega_{1}}$. Take a countable dense set $D$ of $H$ (cf. [1, Theorem 2.3.15]) and let $T=(X[\geq] \times\{\omega\}) \cup(D \times \omega) \subset H \times(\omega+1)$, where $\omega+1$ has the order topology. Topologize $T$ by declaring that a basic neighborhood of a point $\langle t, \omega\rangle \in X[\geq] \times\{\omega\}$ is a neighborhood in the subspace $T$ of the product $H \times(\omega+1)$, and all points of $D \times \omega$ are isolated. Then $T$ satisfies $\left(S^{\prime}\right)$, since it is separable and perfect. On the other hand, $T$ fails to satisfy $(S)$, since it contains a closed subspace $X[\geq] \times\{\omega\}$ which does not satisfy $(S)$.

Acknowledgments. The author would like to thank Professor Haruto Ohta, who communicated to the author the examples showing that the second and third arrows in Remark 3.8 cannot be reversed.

\section{References}

[1] R. Engelking, General Topology, Heldermann, Berlin, 1989.

[2] G. Gruenhage, Generalized metric spaces, in: Handbook of Set-Theoretic Topology, K. Kunen and J. E. Vaughan (eds.), North-Holland, 1984, 423-501.

[3] V. Gutev, H. Ohta and K. Yamazaki, Selections and sandwich-like properties via semi-continuous Banach-valued functions, J. Math. Soc. Japan 55 (2003), 499-521.

[4] R. W. Hansell, On characterizing non-separable analytic and extended Borel sets as types of continuous images, Proc. London Math. Soc. 28 (1974), 683-699.

[5] R. E. Hodel, Spaces defined by sequences of open covers which guarantee that certain sequences have cluster points, Duke Math. J. 39 (1972), 253-263.

[6] R. E. Megginson, An Introduction to Banach Space Theory, Springer, New York, 1998.

[7] E. Michael, Continuous selections I, Ann. of Math. 63 (1956), 361-382.

[8] —, Dense families of continuous selections, Fund. Math. 47 (1959), 173-178.

[9] K. Morita, Products of normal spaces with metric spaces, Math. Ann. 154 (1964), $365-382$.

[10] S. Nedev, Selection and factorization theorems for set-valued mappings, Serdica 6 (1980), 291-317.

[11] C. M. Pareek, Perfect spaces, Questions Answers Gen. Topology 9 (1991), 151-157.

[12] D. Repovš and P. V. Semenov, Continuous Selections of Multivalued Mappings, Kluwer, Dordrecht, 1998.

[13] -, -, Continuous selections of multivalued mappings, in: Recent Progress in General Topology, II, M. Hušek and J. van Mill (eds.), North-Holland, Amsterdam, 2002, 423-461.

[14] M. E. Rudin, A normal space $X$ for which $X \times I$ is not normal, Fund. Math. 73 (1971), 179-186.

[15] J. C. Smith, Properties of expandable spaces, in: General Topology and its Relations to Modern Analysis and Algebra, III (Prague, 1971), J. Novák (ed.), Academia, Prague, 1972, 405-410.

[16] S. Todorčević, A topology on sequences of countable ordinals, Bull. Polish Acad. Sci. Math. 39 (1991), 137-140.

[17] J. M. Worrell, Jr. and H. H. Wicke, Characterizations of developable topological spaces, Canad. J. Math. 17 (1965), 820-830. 
[18] T. Yamauchi, Continuous selections avoiding extreme points, Topology Appl. 155 (2008), 916-922.

[19] —, The role of countable paracompactness for continuous selections avoiding extreme points, preprint.

Takamitsu Yamauchi

Department of Mathematics

Shimane University

Matsue, 690-8504, Japan
Current address:

Department of General Education

Kure College of Technology

Kure, 737-8506, Japan

E-mail: yamauchi@kure-nct.ac.jp

Received December 12, 2007;

received in final form May 31, 2008 